Borek, A., McLaren, B.M., Karabinos, M., \& Yaron, D. (2009). How Much Assistance is Helpful to Students in Discovery Learning? In U. Cress, V. Dimitrova, \& M. Specht (Eds.), Proceedings of the Fourth European Conference on Technology Enhanced Learning, Learning in the Synergy of Multiple Disciplines (EC-TEL 2009), LNCS 5794, September/October 2009, Nice, France. (pp. 391-404). Springer-Verlag Berlin Heidelberg.

\title{
How Much Assistance is Helpful to Students in Discovery Learning?
}

\author{
Alexander Borek ${ }^{12}$, Bruce M. McLaren ${ }^{23}$, Michael Karabinos ${ }^{2}$, David Yaron ${ }^{2}$ \\ ${ }^{1}$ University of Karlsruhe, Germany \\ ${ }^{2}$ Carnegie Mellon University, USA \\ ${ }^{3}$ German Research Center for Artificial Intelligence, Germany \\ alexander.borek@gmail.com, bmclaren@cs.cmu.edu
}

\begin{abstract}
How much help helps in discovery learning? This question is one instance of the assistance dilemma, an important issue in the learning sciences and educational technology research. To explore this question, we conducted a study involving 87 college students solving problems in a virtual chemistry laboratory (VLab), testing three points along an assistance continuum: (1) a minimal assistance, inquiry-learning approach, in which students used the VLab with no hints and minimal feedback; (2) a mid-level assistance, tutored approach, in which students received intelligent tutoring hints and feedback while using the VLab (i.e., help given on request and feedback on incorrect steps); and (3) a high assistance, direct-instruction approach, in which students were coaxed to follow a specific set of steps in the VLab. Although there was no difference in learning results between conditions on near transfer posttest questions, students in the tutored condition did significantly better on conceptual posttest questions than students in the other two conditions. Furthermore, the more advanced students in the tutored condition, those who performed better on a pretest, did significantly better on the conceptual posttest than their counterparts in the other two conditions. Thus, it appears that students in the tutored condition had just the right amount of assistance, and that the better students in that condition used their superior metacognitive skills and/or motivation to decide when to use the available assistance to their best advantage.
\end{abstract}

Keywords: assistance dilemma, intelligent tutoring, inquiry learning, chemistry learning

\section{Introduction}

A key goal of educational technology research is to find the right level of support to imbue in computer-based educational systems. The so-called assistance dilemma is central to this goal: "How should learning environments balance assistance giving and withholding to achieve optimal student learning?" [1]. Assistance giving allows students to move forward when they are struggling and truly need help, yet can rob them of the motivation to learn on their own. On the other hand, assistance withholding encourages students to think and learn for themselves, yet can cause frustration when they are unsure of what to do next. 
Although the "assistance dilemma" is a relatively new term, it describes a central issue in the learning sciences that has been debated for some time. The extreme position of assistance giving is usually called direct-instruction or guided learning. Supporters of this position (e.g. $[2,3,4]$ ) argue that higher assistance (direct instruction and/or tutoring of basic skills) leads to better learning results because it provides information that students cannot create on their own. Supporters of the opposing position (e.g. $[5,6,7,8]$ ) advocate a much lower assistance approach (i.e., assistance withholding), often called discovery or inquiry learning. They argue that assistance withholding lets students construct knowledge on their own.

Other researchers have suggested that the optimal instructional design depends on the students' level of understanding $[9,10]$. For instance, it has been suggested that giving full instructions to novices, and then fading support as the novices' knowledge improves, is best for learning [9]. On the other hand, this work has not precisely identified the amount or timing of assistance that should be provided.

More recently, some researchers have suggested that the assistance dilemma can be viewed along various "dimensions," such as timing of feedback and example study vs. problem solving, and that it may be possible to develop predictive models of the right level of assistance necessary for optimal learning along these dimensions $[11,12]$. In general, this work, while still preliminary, suggests that a midlevel assistance approach is usually optimal. For instance, McLaren, Lim and Koedinger [12] investigated the example-problem dimension of the assistance dilemma in three studies in stoichometry, and a mid-level assistance approach, i.e., alternating worked examples and tutored problems, led to the most efficient learning.

In the work reported in this paper we investigate the optimal amount of assistance in a discovery-oriented domain. In contrast to many domains in which problems are more structured, discovery-learning problems usually involve more open-ended experimentation and thus may require a different level of assistance. Our interest in this work is in taking a first step at identifying the optimal amount of assistance in such discovery learning domains. Our approach focuses on three dimensions of assistance that have been explored in more structured and formalized domains, i.e.:

1) Should immediate yes/no feedback be given to students?

2) What type of feedback content should be given to students?

3) When, how much and what kind of hints should be given to students?

In our experiment we used a virtual chemistry laboratory [13], which we integrated with an intelligent tutor built with the Cognitive Tutoring Authoring Tools (CTAT), an authoring system for cognitive and example-tracing tutors [14]. We tested three widely varying points along a assistance continuum in a real classroom setting: from minimal assistance, in which only very basic feedback was provided, to medium assistance, in which help was given upon request or on incorrect steps, to high assistance, in which students were coaxed to take an optimal problem-solving approach. Our goal was to determine which level of assistance leads to the best learning outcomes in a discovery-learning environment. To our knowledge, there has been no prior study that has compared these three (quite different) levels of assistance in a discovery-learning context. 
A secondary aim of the work was to experiment with CTAT in building intelligent tutors for domains involving simulation and discovery learning. The work we have done and the results we have obtained, although still preliminary, indicate that CTAT can be successfully employed in such a discovery-like domain.

\section{The Technology}

The virtual chemistry laboratory, called the VLab for short, is a computer-based learning environment that simulates an actual chemistry laboratory [13]. The VLab was developed to support introductory level chemistry learning and can be used to perform virtual experiments in various branches of chemistry, such as thermo chemistry and stoichiometry. To design and run experiments, students choose from various tools and substances (e.g. beakers, pipets, flasks, foam cups, bunsen burners, different solutions) (See Fig. 1). The VLab serves as "a bridge between the traditional paper-and-pencil activities from the textbook and actual chemical phenomena" [13] enabling a new kind of interactive learning of chemistry phenomena [15]. The key idea behind the VLab is to help students connect factual and procedural knowledge through authentic chemistry activities, such as experimental design and interpretation.

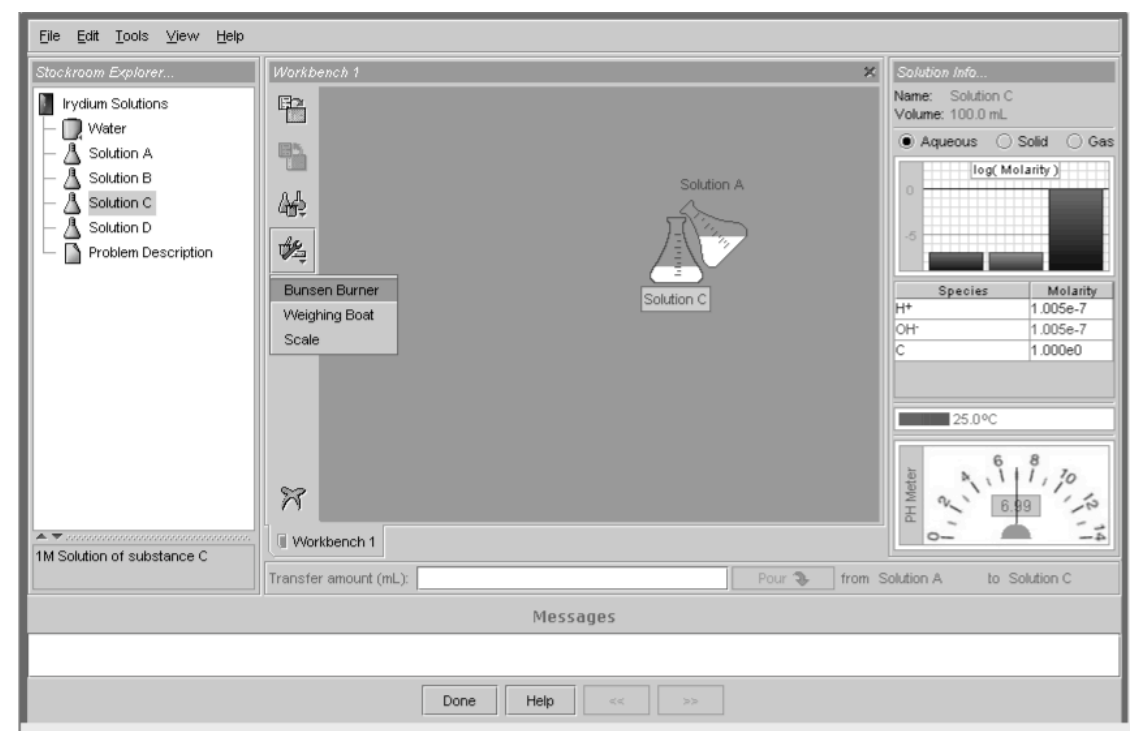

Fig. 1. A screenshot of the VLab as integrated with the Cognitive Tutoring Authoring Tools

In the base version of the VLab no instructions, hints, or feedback messages are provided. To provide such assistance to the student, we integrated the VLab with an intelligent tutor [17] built with CTAT [14]. CTAT supports the capability to build example-tracing tutors, a type of intelligent tutor built using programming-bydemonstration techniques [16]. Example-tracing tutors emulate the behavior of Cognitive Tutors [18], but with lower authoring costs and without the requirement 
of programming skills. Example-tracing tutors work by comparing student problem solving steps to examples of desirable problem-solving behavior.

As part of this project, the "Messages" box, "Done" button, and "Help" button shown at the bottom of Fig. 1 were added to the VLab's user interface, and a CTAT tutor was integrated with VLab. These changes allow the VLab to be more supportive, providing the student with more help and feedback regarding on-going activities than was previously possible. Two of the study conditions utilize this extended version of VLab, as explained in the description of conditions below.

\section{Method}

\subsection{Design}

The study compared three conditions in which students used different versions of the VLab to solve problems in thermo chemistry:

1) the Inquiry-learning Condition, in which students worked with a version of VLab with no hints and minimal feedback,

2) the Tutored Condition, in which students could request hints and received feedback only when they were severely off track ${ }^{1}$, and

3) the Direct-instruction Condition, in which students were directed to follow a prescribed problem-solving path.

Students were given the "discovery" task of mixing chemical solutions that lead to a desired final temperature. This goal was posed in the context of a real world task: preparing food while on a camping trip. The VLab contained solutions of two chemical species, $\mathrm{X}$ and $\mathrm{Y}$, that react to form $\mathrm{Z}$ via the reaction $\mathrm{X}+\mathrm{Y} \rightarrow \mathrm{Z}$. The reaction releases heat that goes into the water and raises the temperature of the solution. The central conceptual basis for solving this problem is the realization that the change in temperature is proportional to the concentration of the initial solutions, where concentration is the number of molecules per unit volume $(1 \mathrm{M}=1$ mole of particles per liter of solution). The student's task was to discover this concept through experimentation with different concentrations.

In the following, we take a closer look at how the assistance differed for each of study conditions as students solved this task (and related subtasks) in the VLab.

Inquiry-learning Condition. This condition used the base version of the VLab. Students were given the general problem description and received no hints, and minimal feedback, on how to solve a particular problem, as outlined in Table 1. The only feedback provided was on the correctness of the final solution (i.e., the concentrations of the solutions mixed together), prompting to continue after completing subtasks, and provision of the final solution if the student reaches an incorrect solution. After solving, students were asked to type an explanation of their observations into a textbox, but no feedback was given on the explanation.

1 One of the authors, David Yaron, is a chemistry professor and provided guidance on diagnosing when a student is "far off track". One example is the obvious misuse of a chemistry tool, e.g., when a bunsen burner is taken from the lab cupboard for a problem not involving the application of heat to substances. 
Table 1. The three dimensions of assistance in the Inquiry-learning Condition.

\begin{tabular}{|l|l|}
\hline $\begin{array}{l}\text { Assistance } \\
\text { Dimensions }\end{array}$ & Inquiry-learning Condition \\
\hline $\begin{array}{l}\text { Immediate } \\
\text { yes/no feed- } \\
\text { back }\end{array}$ & $\begin{array}{l}\text { No immediate yes/no feedback on intermediate steps, but feedback given on } \\
\text { the correctness of the final solution. }\end{array}$ \\
\hline $\begin{array}{l}\text { Feedback } \\
\text { content }\end{array}$ & $\begin{array}{l}\text { Only two types of basic feedback content are provided: } \\
\text { (1) Student is told to move on to the final solution after completing three ex- } \\
\text { planatory tasks (described later), } \\
\text { (2) If student provides incorrect final solution, the correct solution is given. }\end{array}$ \\
\hline $\begin{array}{l}\text { Hint content } \\
\text { and timing }\end{array}$ & No hints available. \\
\hline
\end{tabular}

The Tutored Condition. Students in the tutored condition (see Table 2) were provided with the extended version of the VLab, which used example-tracing CTAT tutors in unordered mode, i.e. students could perform actions in any order. They received no instruction before or after a step unless they explicitly asked for help. By clicking on the help button, the first level of hint appeared, which gave an implicit instruction, for instance, reminding the student of the goal of the current task, e.g. "Your goal is to mix $10 \mathrm{~mL}$ of $1 \mathrm{M} \mathrm{X}$ with $10 \mathrm{~mL}$ of $1 \mathrm{M} \mathrm{Y}$ in a foam cup" or a leading question used to steer the student in the right direction, e.g. "What else do you need to make the solution?" If the first level of hints was not sufficient, students could proceed to the second and third level of hints, which gave gradually more explicit instructions, as shown in Table 2. Students in this condition also received immediate error messages whenever they strayed far off any solution path.

Table 2. The three dimensions of assistance in the Tutored Condition.

\begin{tabular}{|c|c|c|}
\hline $\begin{array}{l}\text { Assistance } \\
\text { Dimensions }\end{array}$ & Tutored Condition & Examples \\
\hline $\begin{array}{l}\text { Immediate } \\
\text { yes/no feed- } \\
\text { back }\end{array}$ & $\begin{array}{l}\text { Immediate feedback on incorrect- } \\
\text { ness only when student is far off } \\
\text { track. No feedback on correct } \\
\text { steps. }\end{array}$ & \\
\hline $\begin{array}{l}\text { Feedback } \\
\text { content }\end{array}$ & $\begin{array}{l}\text { Feedback says which step is } \\
\text { wrong but does not provide ex- } \\
\text { planation why it is wrong. }\end{array}$ & $\begin{array}{l}\text { "No, this was not the right amount of } \\
\text { water." }\end{array}$ \\
\hline $\begin{array}{l}\text { Hint content } \\
\text { and timing }\end{array}$ & $\begin{array}{l}\text { Content: Three levels of hints, } \\
\text { starting with implicit instructions, } \\
\text { gradually becoming more explicit } \\
\text { until specific step is given. } \\
\text { Timing: Each level of hint is gi- } \\
\text { ven upon request only. }\end{array}$ & $\begin{array}{l}\text { Level 1: "What else do you need to } \\
\text { make the solution?" } \\
\text { Level 2: "The } 1 \mathrm{M} \mathrm{X} \text { might be useful." } \\
\text { Level 3: "Click on the flask labeled "1 } \\
\text { M X' in the stockroom and drag it to } \\
\text { the workbench." }\end{array}$ \\
\hline
\end{tabular}


The Direct-instruction Condition. In the direct-instruction condition (see Table 3) students also used the extended version of VLab, but had to follow a specific solution path in a specific step order (ordered mode). A message was given before each step telling the students the precise action they should perform next. Depending on the current step, an explanation of the goal and why the step is sensible was additionally given to the student, e.g. "The goal is to mix $10 \mathrm{~mL}$ of $1 \mathrm{M} \mathrm{X}$ with $10 \mathrm{~mL}$ of $1 \mathrm{M}$ Y." If students did not follow the instruction, even if they took an action on an alternative correct path, an immediate feedback message would be displayed requesting the expected step be taken, e.g., "No, this is wrong. Please reconnect $1 \mathrm{M}$ Reagent $Y$ to the foam cup!" The pedagogical rationale for this form of direct instruction was to ensure that students learn and stay on the optimal solution path.

Table 3. The three dimensions of assistance in the Direct-instruction Condition.

\begin{tabular}{|c|c|c|}
\hline $\begin{array}{l}\text { Assistance } \\
\text { Dimensions }\end{array}$ & Direct-instruction Condition & Examples \\
\hline $\begin{array}{l}\text { Immediate } \\
\text { yes/no feed- } \\
\text { back }\end{array}$ & $\begin{array}{l}\text { Immediate yes/no feedback on } \\
\text { every correct and incorrect step. }\end{array}$ & \\
\hline $\begin{array}{l}\text { Feedback } \\
\text { content }\end{array}$ & $\begin{array}{l}\text { Explicit instructions and expla- } \\
\text { nations for each incorrect step. }\end{array}$ & $\begin{array}{l}\text { "No, this is not correct. Remove this } \\
\text { item from the workbench and take out } \\
\text { the foam cup. A foam cup is better, be- } \\
\text { cause it is insulated and will prevent the } \\
\text { heat generated by the reaction from es- } \\
\text { caping into the surroundings." }\end{array}$ \\
\hline $\begin{array}{l}\text { Hint content } \\
\text { and timing }\end{array}$ & $\begin{array}{l}\text { Content: Explicit instruction be- } \\
\text { fore each action, containing an } \\
\text { explanation of the goal. One } \\
\text { additional explicit hint is also } \\
\text { available upon request, specify- } \\
\text { ing the instruction in more de- } \\
\text { tail. } \\
\text { Timing: Explicit instruction is } \\
\text { given automatically before the } \\
\text { student takes each step. One } \\
\text { additional hint available upon } \\
\text { request only. }\end{array}$ & $\begin{array}{l}\text { Explicit instruction before each action, } \\
\text { given automatically: "The goal is to } \\
\text { mix } 10 \text { mL of } 1 \mathrm{M} \mathrm{X} \text { with } 10 \mathrm{~mL} \text { of } \\
1 \mathrm{M} \mathrm{Y.} \mathrm{To} \mathrm{begin,} \mathrm{select} \mathrm{the} \mathrm{flask} \mathrm{labeled} \\
\text { ' } 1 \mathrm{M} \text { Reagent X' in the stockroom and } \\
\text { drag it to the workbench." } \\
\text { Additional hint upon request: "Take out } \\
\text { the foam cup, which is in the glassware } \\
\text { cabinet, and drag it to the workspace. A } \\
\text { foam cup is used because it is insulated } \\
\text { and will prevent the heat generated by } \\
\text { the reaction from escaping into the sur- } \\
\text { roundings." }\end{array}$ \\
\hline
\end{tabular}

\subsection{Hypothesis}

Our hypothesis was that students would learn most effectively when assistance giving and withholding are balanced, i.e., in the Tutored Condition. 


\subsection{Participants and Condition Assignment}

Participants were 87 undergraduate students in a "Modern Chemistry II" course given during the spring term of 2009 at Carnegie Mellon University (U.S.A.). Most students were in their freshman year and had either science or engineering as a major. The materials were presented to students as an optional exercise, with the score on the activity replacing the lowest of the student's quiz grades. (The average of 10 quizzes counts as $20 \%$ of the final course grade.)

Students were randomly assigned to one of the conditions by pulling a number, either "1," "2," or "3." Altogether there were 47 students in the Inquiry-learning Condition, 16 in the Tutored Condition, and 23 in the Direct-instruction Condition ${ }^{2}$. Each student worked alone on his or her own machine using the version of VLab appropriate to the assigned condition, as described above. All students were unaware of the experimental design and the existence of other conditions.

Table 4. Activities during the study

\begin{tabular}{|c|c|c|c|}
\hline Activity & Time & Medium & $\begin{array}{l}\text { Same in all } \\
\text { conditions }\end{array}$ \\
\hline Introduction and Consent & $4 \min$ & Instructor + Paper-based & $\mathrm{Yes}^{3}$ \\
\hline Pretest & $6 \mathrm{~min}$ & Paper-based & Yes \\
\hline $\begin{array}{l}\text { Intervention (different per } \\
\text { condition): Camping Prob- } \\
\text { lem in the VLab }\end{array}$ & $40 \mathrm{~min}$ & Computer-based (VLab) & No \\
\hline Posttest \& Questionnaire & $10 \mathrm{~min}$ & Paper-based & Yes \\
\hline TOTAL & $60 \mathrm{~min}$ & & \\
\hline
\end{tabular}

\subsection{Procedure and Materials}

The study consisted of four basic activities, as shown in Table 4. First, students received a general introduction and a consent form. Second, students took a sixminute paper-based pretest. Third, in the intervention part of the study, students in the three conditions had to solve problems in the VLab according to the different conditions discussed above. They were allotted 40 minutes for this part of the study. Finally, all students completed the paper-based posttest, which was followed by a short questionnaire; they were allowed 10 minutes to complete this portion of

2 Although 150 students actually participated, technical problems led to the elimination of 63 students from the Tutored and Direct-instruction Conditions. Note that while this elimination of subjects led to lower numbers in the Tutored and Direct-instruction Conditions, it did not alter the random nature of assignment or adversely impact the results.

3 Except that the URL to the problem website was given in the Inquiry-learning Condition. 
the study. The entire study took 60 minutes. In the following, a short overview of each activity is provided.

\section{Introduction and Consent}

Before the study started students were asked to read a document describing the study. They were then allowed to decide whether or not to participate. No students elected not to participate.

\section{Pretest}

The study began with a pretest, which was the same for all conditions. The pretest consisted of a reaction equation and an example reaction; students were asked to solve four tasks on their own based on these items in six minutes. These questions probed the direct proportionality between the change in temperature and the enthalpy of reaction (an underlying concept covered in the course lectures) and the direct proportionality between the change in temperature and solution concentration (a concept that had never been explicitly discussed in the course).

\section{Intervention: VLab “Camping Problem”}

Next, the students were presented with the "Camping problem" (see Table 5) and a paper explaining how to use the VLab. They then worked on the "Camping Problem" in the VLab, which differed for each condition according the level of assistance provided, as described above. In each condition, the intervention began with an exploratory phase designed to focus student attention on the relationship between the change in temperature and the concentrations. In the exploratory phase, students were asked to make the following mixtures and measure the resulting change in temperature (as shown in Table 5):

$$
\begin{aligned}
& \text { Mixture A: } 10 \mathrm{~mL} 1.0 \mathrm{M} \mathrm{X}+10 \mathrm{~mL} 1.0 \mathrm{M} \mathrm{Y} \\
& \text { Mixture B: } 5 \mathrm{~mL} 1.0 \mathrm{M} \mathrm{X}+5 \mathrm{~mL} 1.0 \mathrm{M} \mathrm{Y} \\
& \text { Mixture C: } 10 \mathrm{~mL} 0.5 \mathrm{M} \mathrm{X}+10 \mathrm{~mL} 0.5 \mathrm{M} \mathrm{Y}
\end{aligned}
$$

Mixtures A and B lead to identical final temperatures. Reducing the volume by one-half also reduces by one-half the amount of $\mathrm{X}$ and $\mathrm{Y}$ that react and thus halves the amount of heat generated. However, the amount of water that must be heated is also halved such that the final temperature is the same for mixtures $\mathrm{A}$ and $\mathrm{B}$. For mixture $\mathrm{C}$, the temperature change is half that of mixture $\mathrm{A}$, since the amount of $\mathrm{X}$ and $\mathrm{Y}$ that react are cut in half while keeping the amount of water to be heated fixed. Since halving the concentration halves the temperature change, students should infer that the temperature change is proportional to the concentration. This direct proportionality may then be used to determine the concentration required to reach the target temperature.

Following this exploratory phase, students were given the task of creating solutions that would give the desired final temperature for the "Camping Problem." In the Inquiry-learning Condition, only the problem was presented and the students had to find solutions on their own. In contrast, students in the Tutored Condition 
and Direct-instruction Condition were guided through the camping problem by four subtasks (see Table 5: Solution approaches 1 and 2 a,b,c), which demonstrate two different problem approaches. In solution approach 1 , the proportionality relation was used to explicitly calculate the concentration needed to achieve the target concentration. Creating solutions of the desired concentrations required an additional set of dilution computations. In solution approach 2, explicit mathematical computations were avoided by designing experiments that achieve the same goal. First, equal volumes of the starting $\mathrm{X}$ and $\mathrm{Y}$ solutions (1 $\mathrm{M}$ each) were mixed together, leading to a solution that exceeded the target temperature. Water was then added until the temperature reduced to the target temperature. This amount of water was then used to determine the required concentrations.

Table 5. Intervention: "Camping Problem" in the VLab.

Problem Name Short problem description

Explanatory Task $1 \quad$ Mixture A: Mix $10 \mathrm{~mL} 1 \mathrm{M}$ Reagent X with $10 \mathrm{~mL} 1 \mathrm{M}$ Reagent $\mathrm{Y}$ in a foam cup. Type in the change in temperature of the created solution.

Explanatory Task $2 \quad$ Mixture B: Mix $5 \mathrm{~mL} 1 \mathrm{M}$ Reagent X with $5 \mathrm{~mL} 1 \mathrm{M}$ Reagent $\mathrm{Y}$ in a foam cup. Type in the change in temperature of the created solution.

Explanatory Task $3 \quad$ Mixture C: Mix $10 \mathrm{~mL} 0.5$ M Reagent X with $10 \mathrm{~mL} 0.5 \mathrm{M}$ Reagent $\mathrm{Y}$ in a foam cup. Type in the change in temperature of the created solution.

Solution Approach $1^{4} \quad$ Create a solution with the given temperature by calculating the needed concentration and creating those concentration by diluting $\mathrm{X}$ and $\mathrm{Y}$.

Solution Approach $2 a$

Mix $5 \mathrm{~mL} 1 \mathrm{M}$ Reagent $\mathrm{X}$ with $5 \mathrm{~mL} 1 \mathrm{M}$ Reagent $\mathrm{Y}$ in a foam cup. Add $10 \mathrm{~mL}$ of water. Compare the change in temperature of the created solution to that of mixture $\mathrm{C}$, to show that order (dilution before versus after mixture) does not matter.

Solution Approach $2 b \quad$ Mix $10 \mathrm{~mL}$ of $1 \mathrm{M}$ Reagent $\mathrm{X}$ with $10 \mathrm{~mL}$ of $1 \mathrm{M}$ Reagent $\mathrm{Y}$ in a foam cup. Add water until you have reached the desired temperature.

Solution Approach 2c $\quad$ Divide the amount of added water through two and dilute $10 \mathrm{~mL}$ $\mathrm{M}$ Reagent $\mathrm{X}$ and $10 \mathrm{~mL} 1 \mathrm{M}$ Reagent $\mathrm{Y}$ with this amount of water to create the needed concentration. Mix both solutions in a foam cup and type in the resulting change in temperature.

4 Students in the Inquiry-learning Condition were asked to try different solution approaches on their own instead of having to solve solution approaches 1 and 2 a,b,c explicitly. 


\section{Posttest and Questionnaire}

The study concluded with a paper-based posttest, which contained a neartransfer component with standard exercises and a conceptual-understanding component.

The near-transfer part was subdivided into Task 1, which was a collection of several multiple-choice questions, and Task 2, in which students had to use the proportionality of temperature change to the concentration for a calculation. The near-transfer portion of the posttest probed the student's understanding of the direct proportionality between temperature change and solution concentration.

The conceptual-understanding portion of the posttest consisted of two items for which responses were given as free-form text. In the first item, students were asked to write a general design strategy for how to create a solution with a desired temperature. The second item restated the goal of the activity (heating food while on a camping trip) and asked students to list the factors of this approach that would limit meeting this goal. The responses were coded on a rubric that assigned points to each of the factors listed.

At the end of the posttest, but within the allotted 10 minutes, students answered a brief questionnaire, containing six Likert-scale questions (1-5 scale), probing for students' self-assessment on task difficulty, frustration level, usefulness, etc. of the materials, and a single free-form "Comments" section.

\subsection{Results}

We first scored and ran an ANOVA on students' pretests, to assure equality between conditions, with conditions as a between-subjects factor. Tasks had only one acceptable solution and were graded by a program. As there was no significant difference in the pretest between the three conditions, $F(2,77)=0.292, p=.748$, we assume that students in the three conditions started with a similar level of knowledge.

Next, we evaluated the posttest scores. Tasks in the near-transfer part of the posttest also had only one acceptable solution and were scored by a program. Three reviewers (i.e., authors 1,3 , and 4 of this paper) graded the conceptualunderstanding tasks of the posttest, answered in free-form text, using the same rubric to ensure objectivity. In approximately $90 \%$ of cases there was agreement by at least two graders, in the other $10 \%$ the average of all three grades was taken. We removed seven outliers from the population - students who scored less than a quarter of the maximal reachable points in the posttest. Fig. 2 shows the means of the overall posttest scores, as well as the means of the individual components of the posttest (i.e., the near-transfer scores and conceptual-understanding scores).

We then ran ANCOVAs on the posttest scores, using the pretest scores as the covariate, to evaluate differences in the posttest scores between the conditions. Although the mean scores were higher in the Tutored Condition for both the overall score and the near-transfer score, the differences were not significant, $\mathrm{F}(2,77)=2.035, \mathrm{p}=.138 ; \mathrm{F}(2,77)=0.057, \mathrm{p}=.944$. However, we did find a significant result on the conceptual-understanding part of the posttest: Students in the Tutored 
Condition did better on conceptual-understanding tasks than students in the other two conditions, $F(2,77)=3.783, p=.007$. These results support our hypothesis: Students in the Tutored Condition - the mid-level assistance approach - showed better learning results than students in the other two conditions.

\begin{tabular}{|c|c|c|c|}
\hline 0.7 & & & \\
\hline 0.6 & & & \\
\hline 0.5 & & & \\
\hline & & & \\
\hline 0.3 & & & \\
\hline 0.2 & & & \\
\hline 0.1 & & & \\
\hline 0 & $\begin{array}{c}\text { Posttest Overall } \\
\text { Score }\end{array}$ & $\begin{array}{l}\text { Posttest Near- } \\
\text { transfer }\end{array}$ & $\begin{array}{c}\text { Posttest } \\
\text { Conceptual- } \\
\text { understanding }\end{array}$ \\
\hline Inquiry & 0.54 & 0.62 & 0.47 \\
\hline Tutored & 0.62 & 0.63 & 0.62 \\
\hline Direct & 0.57 & 0.6 & 0.54 \\
\hline
\end{tabular}

Fig. 2. Means of the posttest scores across all conditions.

Finally, we segmented students into strong (best 50\%) and weak (worst 50\%) groups based on their pretest scores. In another ANCOVA, again using pretest scores as the covariate, students in the Tutored Condition who did better on the pretest benefitted more regarding conceptual understanding than students in the other conditions, $\mathrm{F}(2,37)=4.699, \mathrm{p}=.015$. Weaker students in the Tutored Condition also did better on the conceptual-understanding part than weaker students in the other conditions, but not significantly, $F(2,37)=1.193$, $p=.315$.

\section{Discussion}

In summary, we observed differences between the three conditions in conceptual understanding, where students in the Tutored Condition scored higher than students in the other conditions. In addition, stronger students in the Tutored Condition had better results than stronger students in the other conditions on the conceptual questions.

So why did students in the Tutored Condition achieve greater conceptual understanding? One possible explanation is that the tutored students were able to make more active decisions, leading to higher motivation. At the same time, they received help when they needed it, which may have prevented frustration. Both of these aspects may, in turn, have led to more learning. In contrast, students in the Direct-instruction Condition may have been demotivated, unable to make their own decisions; that is, they may have received too much assistance for learning. This 
was hinted at by some comments in the feedback questionnaire, e.g. "I disliked having to follow the instructions. It's like communist chemistry." Students in the Inquiry-learning Condition, on the other hand, may have gotten frustrated when they did not know what to do and did not work as hard at learning; that is, they may have received too little assistance. This was suggested by some feedback in the questionnaire, e.g., "It makes me feel really stupid." Both of these comments are consistent with our classroom observation of the students in the two conditions ${ }^{5}$.

It is possible that students in the Direct-instruction Condition were hurt by too little instruction on how to use their version of the VLab. As the study time was very short, an introduction on how to use the CTAT-enabled VLab was given on paper instead of through trial use of the software. Some students in the Directinstruction Condition may not have understood they were supposed to explicitly follow the instructions. In the Inquiry-learning Condition, lack of information may have led to extraneous load [19]. That is, there may have been insufficient cognitive resources available for learning, given the variety of tasks the students had to do simultaneously (i.e., trying to solve the problem, navigate an unfamiliar environment, choose the next substance, etc., all without guidance), thus explaining the lower learning outcomes compared to the Tutored Condition.

Furthermore, as discussed above, the differences in conceptual learning were larger and significant for stronger students than weaker students compared to other conditions. We have two possible interpretations for this finding. First, stronger students are likely to have a higher metacognitive awareness than weaker students [20] and thus may have used the available hints and feedback of the Tutored Condition more effectively. Second, stronger students, who tend to be more independent learners, may have simply been more motivated to learn since they were allowed to make their own decisions and construct their own knowledge, asking for help only when they really felt they needed it.

Finally, why were differences only observed for conceptual questions? This can be explained by the nature of the camping problem, which is focused on conceptual aspects of thermo chemistry. That is, the camping problem, and use of the VLab to solve it, focused students on running experiments to learn concepts, rather than procedures or calculations. The procedure and calculations necessary to solve the near-transfer problems were done outside of the VLab in all conditions; thus, we would not (necessarily) expect that any of the conditions would do better than the others in the near-transfer part of the posttest.

\section{Conclusion}

The assistance dilemma is concerned with the subtle choices involved in offering assistance to students as they engage in problem-solving activities and how to make choices that will optimize learning. The assistance dilemma becomes especially cogent when students engage with a software environment, such as the chemistry VLab, focused on inquiry and discovery. By integrating the VLab with an in-

5 Unfortunately we were unable to analyze these, and other possible explanations, through process analysis, since all logging data was lost due to a technical problem. 
telligent tutor, we were able to experiment with different levels of assistance, varied along different dimensions (i.e., timing, content, and type of feedback and hints).

The study presented in this paper was conducted in a real science classroom setting using three conditions that span the assistance continuum of discovery learning. We found that students in a Tutored Condition (mid-level assistance) learned better on conceptual tasks than students in both a greater- and lesser-assistance condition. That is, it appears that the Tutored Condition provided the best balance of giving and withholding assistance. Moreover, stronger students benefited more from the Tutored Condition than weaker students. The results support the notion that the optimal level of assistance lies between the extremes of direct instruction and pure discovery, and that the learning gains from a given level of assistance vary based on student characteristics, such as student pre-knowledge. Furthermore, the results suggest that assistance should be given only when students are far off track or in response to student requests for help (as opposed to being offered immediately at each step, as in the direct instruction condition).

On the other hand, our study was of a limited duration (60 minutes), with a single student pool in a single domain of science, and did not include any measure of long-term retention (sometimes argued as the only real learning measure [21]). In addition, a process analysis of student activities during the intervention was not possible due to technical problems. Log data may have revealed how students utilize assistance, as well as the origins of the learning effects.

Nevertheless, the work presented here, i.e., the merging of an open-ended discovery-learning environment with an intelligent tutor and achieving hypothesized learning results in a controlled study with variations on this system, is encouraging. We intend to replicate this experiment in more chemistry classrooms during the next school year. In addition, we believe the results may extend to other areas of science in which discovery learning is often used (e.g., "discovering" Darwin's theory of evolution) and intend to apply this experimental model in such domains. In sum, this research represents an important step towards our long-term goal of developing a predictive model for the optimal amount of assistance to provide to students as they engage in a range of authentic learning activities.

Acknowledgments. The Pittsburgh Science of Learning Center (PSLC), NSF Grant \# 0354420 provided support for this research.

\section{References}

[1] Koedinger, K.R., Aleven, V.: Exploring the Assistance Dilemma in Experiments with Cognitive Tutors. Educational Psychology Review 19, 239-264 (2007)

[2] Klahr, D., Nigam, M.: The Equivalence of Learning Paths in Early Science Instruction - Effects of Direct Instruction and Discovery Learning. Psychological Science, 661667 (2004)

[3] Mayer, R.E.: Should There Be a Three-Strikes Rule Against Pure Discovery Learning? - The Case for Guided Methods of Instruction. American Psychologist, 14-19 (2004)

[4] Kirschner, P.A., Sweller, J., Clark, R.E.: Why Minimal Guidance During Instruction Does Not Work: An Analysis of the Failure of Constructivist, Discovery, Problem- 
Based, Experiential, and Inquiry-Based Teaching. Educational Psychologist, 75-86 (2006)

[5] Bruner, J.S.: The Art of Discovery. Harvard Educational Review (31), 21 - 32 (1961)

[6] Barrows, H.S., Tamblyn, R.M.: Problem-based Learning: An Approach to Medical Education. New York: Springer. (1980)

[7] Jonassen, D.: Objectivism vs. Constructivism. Educational Technology Research and Development, 39(3), 5-14 (1991)

[8] Steffe, L., Gale, J.: Constructivism in Education. Hillsdale, NJ: Lawrence Erlbaum Associates, Inc. (1995)

[9] Renkl, A., Atkinson, R.K., Große, C.S.: How Fading Worked Solution Steps Works A Cognitive Load Perspective. Instructional Science, 32, 59-82. (2004)

[10] Cronbach, L., Snow, R.: Aptitudes and Instructional Methods: A Handbook for Research on Interactions. New York: Irvington Publishers. (1977)

[11] Koedinger, K.R., Pavlik Jr., P.I., McLaren, B.M., Aleven, V.: Is it Better to Give than to Receive? The Assistance Dilemma as a Fundamental Unsolved Problem in the Cognitive Science of Learning and Instruction. In: B. C. Love, K. McRae, \& V. M. Sloutsky (Ed.), Proceedings of the 30th Annual Conference of the Cognitive Science Society pp. 2155-2160. Austin, TX: Cognitive Science Society. (2008)

[12] McLaren, B.M., Lim, S., Koedinger, K.R.: When and How Often Should Worked Examples be Given to Students? New Results and a Summary of the Current State of Research. In: B. C. Love, K. McRae, \& V. M. Sloutsky (Ed.), Proceedings of the 30th Annual Conference of the Cognitive Science Society pp. 2176-2181 Austin, TX: Cognitive Science Society. (2008)

[13] Yaron, D., Evans, K., Karabinos, M.: Scenes and Labs Supporting Online Chemistry. Paper presented at the $83^{\text {rd }}$ Annual AERA National Conference (2003)

[14] Aleven, V., McLaren, B.M., Sewall, J., Koedinger, K.R.: Example-Tracing Tutors: A New Paradigm for Intelligent Tutoring Systems. International Journal of Artificial Intelligence in Education (IJAIED), Special Issue on "Authoring Systems for Intelligent Tutoring Systems." (2009)

[15] Yaron, D., Freeland, R., Lange, D., Milton, J.: Using Simulations to Transform the Nature of Chemistry Homework. CONFCHEM (CONFerences on CHEMistry): On-Line Teaching Methods. http://www.ched-ccce.org/confchem/ Online-Conference: American Chemical Society. (2000)

[16] Lieberman, H. (Ed.): Your Wish is My Command: Programming by Example. Morgan Kaufmann, San Francisco, CA. (2001)

[17] VanLehn, K.: The Behavior of Tutoring Systems. International Journal of Artificial Intelligence in Education (IJAIED), 16, $227-265$ (2006)

[18] Koedinger, K.R., Anderson, J.R., Hadley, W.H., Mark, M.A.: Intelligent tutoring goes to school in the big city. International Journal of Artificial Intelligence in Education (IJAIED), 8, 30-43 (1997)

[19] Sweller, J., Van Merriënboer, J.J.G., Paas, F.G.W.C.: Cognitive Architecture and Instructional Design. Educational Psychology Review, 10, 251-296. (1998)

[20] Bransford, J.D., Brown, A.L., Cocking, R.R. (Eds.) How People Learn: Brain, Mind, Experience, and School. Washington, CD: National Academy Press. (2000)

[21] Schmidt, R.A., Bjork, R.A.: New Conceptualizations of Practice: Common Principles in Three Paradigms Suggest New Concepts for Training. Psychological Science, 3(4), 207-217. (1992) 